\title{
Pharmacokinetics of Coadministered Guanfacine Extended Release and Lisdexamfetamine Dimesylate
}

\author{
Benno Roesch • Mary E. Corcoran · Jennifer Fetterolf • \\ Mary Haffey • Patrick Martin • Peter Preston • \\ Jaideep Purkayastha $\cdot$ Phillip Wang $\cdot$ James Ermer
}

Published online: 25 April 2013

(C) The Author(s) 2013. This article is published with open access at Springerlink.com

\begin{abstract}
Background In clinical practice, $\alpha_{2}$-adrenoceptor agonists have been adjunctively administered with psychostimulants for the treatment of attention-deficit/hyperactivity disorder (ADHD). Two studies have examined the adjunctive use of guanfacine extended release (GXR, Intuniv ${ }^{\circledR}$; Shire Development LLC, Wayne, PA, USA) with psychostimulants in children and adolescents with a suboptimal response to psychostimulant treatment. However, the potential for pharmacokinetic drug-drug interactions (DDIs) between GXR and lisdexamfetamine dimesylate (LDX, Vyvanse ${ }^{\circledR}$; Shire US LLC, Wayne, PA, USA) has not been thoroughly evaluated.

Objective The primary objective of this study was to examine the pharmacokinetics of GXR $4 \mathrm{mg}$ and LDX $50 \mathrm{mg}$ given as single doses alone and in combination.

Study Design This was an open-label, randomized, threeperiod crossover, DDI study.

Setting The study was conducted in a single clinical research center.

Participants Forty-two healthy adults were randomized in this study.
\end{abstract}

M. Haffey: deceased.

Clinical trial registration number (ClinicalTrials.gov): NCT00919867.

B. Roesch ( $\square)$

Advanced Biomedical Research, Inc., 241 Main Street,

3rd Floor, Hackensack, NJ 07601, USA

e-mail: bennoro@cs.com

M. E. Corcoran · J. Fetterolf · M. Haffey · P. Martin ·

P. Preston - J. Purkayastha $\cdot$ P. Wang - J. Ermer

Shire Development LLC, Wayne, PA, USA
Interventions Subjects were administered single oral doses of GXR $4 \mathrm{mg}$, LDX $50 \mathrm{mg}$, or GXR and LDX in combination.

Main Outcome Measures Blood samples collected predose and up to $72 \mathrm{~h}$ postdose assessed guanfacine, LDX, and $d$-amphetamine levels. Bioequivalence was defined as the $90 \%$ confidence intervals (CIs) of the geometric mean ratios of the area under the plasma concentration-time curve extrapolated to infinity $\left(\mathrm{AUC}_{0-\infty}\right)$ and maximum plasma concentration $\left(C_{\max }\right)$ falling within the bioequivalence reference interval ( $0.80-1.25)$. Safety measures included adverse events, vital signs, and electrocardiograms (ECGs).

Results Forty subjects completed the study. Following administration of LDX alone or in combination with GXR, the statistical comparisons of the $\mathrm{AUC}_{0-\infty}$ and $C_{\max }$ of $d$ amphetamine fell entirely within the reference interval. For guanfacine, the $90 \% \mathrm{CI}$ of the geometric mean ratio of $\mathrm{AUC}_{\infty}$ for the two treatments was within the bioequivalence criteria, but for $C_{\max }$ the upper bound of the $90 \% \mathrm{CI}$ exceeded the standard range for bioequivalence by $7 \%$. This relatively small change is unlikely to be clinically meaningful. Treatment-emergent adverse events (TEAEs) were reported by $42.9 \%$ of subjects; the most commonly reported TEAEs included dizziness $(5.0,7.3$, and $7.3 \%$ ) and headache $(7.5,4.9$, and $7.3 \%)$ following administration of GXR, LDX, and GXR and LDX in combination, respectively. Clinically significant ECG abnormalities occurred in one subject following administration of LDX and in one subject following coadministration of GXR and LDX.

Conclusions In healthy adults, coadministration of GXR and LDX did not result in a clinically meaningful pharmacokinetic DDI compared with either treatment alone. No unique TEAEs were observed with coadministration of GXR and LDX compared with either treatment alone. 


\section{Introduction}

In clinical practice, $\alpha_{2}$-adrenoceptor agonists have been adjunctively administered with psychostimulants for the treatment of attention-deficit/hyperactivity disorder (ADHD) [1-4]. Guanfacine extended release (GXR; Intuniv ${ }^{\circledR}$; Shire Development Inc., Wayne, PA, USA), a selective $\alpha_{2 \mathrm{~A}}$-adrenoceptor agonist [5], is approved by the US Food and Drug Administration as monotherapy and as adjunctive therapy to psychostimulant medications for the treatment of ADHD in children and adolescents aged 6-17 years [5]. Treatment-emergent adverse events (TEAEs) commonly reported with GXR monotherapy treatment include somnolence, fatigue, nausea, lethargy, and hypotension [6-10]. Patients taking GXR have demonstrated similar growth compared with normative data [5].

Psychostimulants are the most widely prescribed pharmacologic agents for the treatment of ADHD [11, 12]. Lisdexamfetamine dimesylate (LDX; Vyvanse ${ }^{\circledR}$; Shire US LLC, Wayne, PA, USA) is a long-acting prodrug psychostimulant, which is approved as monotherapy for the treatment of ADHD in children (aged 6-12 years), in adolescents (aged 13-17 years), and in adults [13]. TEAEs commonly reported with LDX treatment across these populations include anxiety, decreased appetite, diarrhea, dry mouth, insomnia, irritability, nausea, upper abdominal pain, and vomiting [13].

Two studies have examined the adjunctive use of GXR with psychostimulants in children and adolescents with a suboptimal response to psychostimulant treatment. An open-label study found that the addition of GXR did not result in unique adverse events (AEs) compared with those reported historically with either treatment alone, and adjunctive administration was also associated with significant improvements in ADHD symptoms [14]. The results of this earlier study were confirmed in a large, pivotal, multicenter, randomized, placebo-controlled study of GXR adjunctive to psychostimulants [15]. Despite these earlier investigations, the potential for pharmacokinetic drug-drug interactions (DDIs) between GXR and LDX has not been thoroughly evaluated.

Pharmacokinetic DDIs can occur when two medications are coadministered, resulting in a change in the metabolism, absorption, tissue and/or plasma binding, distribution, or elimination of one or both medications [16]. Although guanfacine is known to be metabolized by cytochrome P450 (CYP) 3A4 [5], LDX is absorbed as the intact prodrug and is converted via enzymatic hydrolysis to $l$-lysine and therapeutically active $d$-amphetamine primarily in the blood by red blood cells [17]. Although intact LDX is not metabolized by the CYP system and is neither an inducer nor an inhibitor of the system, the metabolism of $d$ amphetamine has not been fully characterized $[13,18]$. It is therefore prudent to study the pharmacokinetics of GXR coadministered with LDX to confirm the lack of metabolic interactions between these two therapies.

Although there is a lack of pharmacokinetic data on coadministration of GXR and LDX, pharmacokinetic studies of each medication administered alone have been published [19-24]. An open-label, dose-escalation, pharmacokinetic study of GXR in children (aged 6-12 years) and adolescents (aged 13-17 years) with ADHD showed that GXR exhibits a linear pharmacokinetic profile [19]. A linear pharmacokinetic profile of GXR was also observed in an open-label crossover study examining single doses of GXR 1-, 2-, and 4-mg tablets in healthy adults aged 18-55 years [20]. Maximum guanfacine concentrations of $0.98,1.57$, and $3.58 \mathrm{ng} / \mathrm{mL}$ were attained at $6 \mathrm{~h}$ for the $1-$ and $2-\mathrm{mg}$ doses and at $5.5 \mathrm{~h}$ for $4-\mathrm{mg}$ doses. When administered alone, LDX has demonstrated a linear dose-proportional pharmacokinetic profile in both children and adults [21, 22]. Maximum mean $d$-amphetamine concentrations of 53.2, 93.3, and $134 \mathrm{ng} / \mathrm{mL}$ were attained in children with ADHD at $3.5 \mathrm{~h}$ for the 30-, 50-, and 70-mg doses, respectively [21]. In healthy adults, maximum mean $d$-amphetamine concentrations of $44.6,84.6$, and $126.6 \mathrm{ng} / \mathrm{mL}$ were attained at $4 \mathrm{~h}$ for the 50-, 100-, and 150-mg doses. For the 200- and 250-mg doses, maximum mean concentrations of 168.8

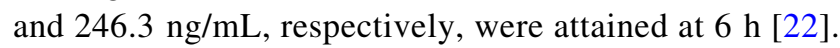
Two studies that assessed the pharmacokinetics of LDX $70 \mathrm{mg}$ in healthy adults found maximum mean $d$-amphetamine concentrations of 80.3 and $90.1 \mathrm{ng} / \mathrm{mL}$ at $3 \mathrm{~h}[23,24]$.

The safety profiles of GXR and LDX have been examined in previous studies. In subjects who received GXR in clinical trials, systolic blood pressure (SBP), diastolic blood pressure (DBP), and pulse rate decreased in a dosedependent manner $[6,7,9]$. Mean increases in SBP, DBP (2-4 $\mathrm{mmHg}$ ), and pulse rate $(3-6$ beats $/ \mathrm{min})$ are often reported with LDX treatment [14, 25, 26].

The primary purpose of this present study was to evaluate the pharmacokinetic profiles of GXR and LDX, administered alone and in combination, in healthy adults. Evaluating the safety of GXR, LDX, and coadministered GXR and LDX was a secondary objective of the study.

\section{Materials and Methods}

This was an open-label, randomized, three-period DDI study of GXR and LDX in healthy adults aged 18-45 years. Written informed consent was obtained from each subject, in accordance with the International Conference on Harmonisation (ICH) Good Clinical Practice (GCP) Guideline E6 and applicable regulations. 
At screening, the inclusion criteria were a body mass index between 20.0 and $30.0 \mathrm{~kg} / \mathrm{m}^{2}$ (inclusive); a satisfactory medical assessment with no significant or relevant abnormalities in medical history, physical examination, or vital signs; no laboratory evaluation that was considered reasonably likely to interfere with the subject's participation in or ability to complete the study; and normal or clinically insignificant electrocardiogram (ECG) findings at screening. Subjects were excluded from the study if they had current or recurrent disease that could affect clinical or laboratory assessments; a history of seizure disorder; a history or presence of known cardiac abnormalities, syncope, cardiac conduction problems, exerciserelated cardiac events, or clinically significant bradycardia; a history of controlled or uncontrolled hypertension or a resting sitting SBP greater than $139 \mathrm{mmHg}$ or DBP greater than $89 \mathrm{mmHg}$; and symptomatic or clinically meaningful orthostatic hypotension as assessed by the investigator.

On day 1 of the first treatment period, subjects were randomly assigned to one of the six possible treatment sequences (i.e., $\mathrm{ABC}, \mathrm{ACB}, \mathrm{BAC}, \mathrm{BCA}, \mathrm{CAB}, \mathrm{CBA}$ ) (Fig. 1). During each of the study's three treatment periods, subjects were administered one of three medication regimens: regimen A consisted of a single 4-mg dose of GXR; regimen $\mathrm{B}$ consisted of a single $50-\mathrm{mg}$ dose of LDX; regimen $C$ consisted of coadministration of single doses of GXR (4 mg) and LDX (50 mg). Subjects were confined to the clinical research center during each treatment period (i.e., from day -1 through day 4). The total confinement for this study was 12 days. Washout periods of at least 7 days separated the treatment periods.

\subsection{Pharmacokinetic Assessments}

Guanfacine, lisdexamfetamine, and $d$-amphetamine levels were measured in plasma produced from blood samples collected at predose (within $30 \mathrm{~min}$ of administration) and at $0.5,1.0,1.5,2.0,3.0,4.0,6.0,8.0,12,24,30,48$, and $72 \mathrm{~h}$ after treatment. Blood samples were centrifuged at approximately $2,500 \mathrm{rpm}$ for $15 \mathrm{~min}$ at $4{ }^{\circ} \mathrm{C}$ within $30 \mathrm{~min}$ of the blood draw. Plasma concentrations were measured using liquid chromatography (LC) with tandem mass spectrometry (LC-MS/MS) methods that were validated for the quantitation of guanfacine, lisdexamfetamine, and $d$-amphetamine in human $\mathrm{K}_{3}$-EDTA plasma.

For guanfacine, the LC-MS/MS analysis was carried out with a Sciex 4000 mass spectrometer coupled with a Shimadzu LC pump (model LC-10AT) and Perkin-Elmer 200 series autosampler. The internal standard used was guanfacine $\left({ }^{13} \mathrm{C}^{15} \mathrm{~N}_{3}\right)$. Guanfacine and its internal standard were extracted from $200 \mu \mathrm{L}$ of human plasma by liquid-liquid extraction prior to LC-MS/MS analysis. The chromatographic separation was achieved on an XBridge phenyl, $3.5 \mu \mathrm{m}, 4.60 \times 50 \mathrm{~mm}$ LC column (Waters Corporation), with mobile phase at a flow rate of $1 \mathrm{~mL} / \mathrm{min}$. The mass spectrometer was operated in positive electrospray ionization mode, and the resolution settings used were unit for Q1 and low for Q3. The multiple reaction monitoring (MRM) transition was $m / z 246 \rightarrow 60$ for guanfacine, and the MRM transition was $m / z, 250 \rightarrow 159$ for the internal standard, guanfacine $\left({ }^{13} \mathrm{C}^{15} \mathrm{~N}_{3}\right)$. On the basis of a sample volume of $200 \mu \mathrm{L}$, the assay ranged from 0.05 to $50 \mathrm{ng} / \mathrm{mL}$ for guanfacine. Samples over the limit of quantitation were diluted into range with control plasma.

For $d$-amphetamine and lisdexamfetamine, the LC-MS/ MS analysis was carried out with a Sciex API 3000 mass spectrometer coupled with a Shimadzu LC pump (model LC-10AT) and Perkin-Elmer 200 series autosampler. The internal standards used were amphetamine- $\mathrm{D}_{5}$ for $d$ amphetamine and lisdexamfetamine- $\mathrm{D}_{8}$ for lisdexamfetamine. Plasma samples containing $d$-amphetamine, lisdexamfetamine, and their internal standards were extracted by liquid-liquid extraction prior to the LC-MS/MS analysis. The chromatographic separation was achieved on a Phenosphere NEXT CN, $5 \mu \mathrm{m}, 4.6 \times 50 \mathrm{~mm}$ column (Phenomenex), with mobile phase at a flow rate of $1 \mathrm{~mL} /$ min. The mass spectrometer was operated in positive mode, and the resolution setting used was unit for both Q1 and Q3. The MRM transitions were $m / z 136 \rightarrow 91$ for $d$ amphetamine, $m / z \quad 141 \rightarrow 96$ for amphetamine- $\mathrm{D}_{5}$, $m / z 264 \rightarrow 84$ for lisdexamfetamine, and $m / z \quad 272 \rightarrow 92$ for lisdexamfetamine- $\mathrm{D}_{8}$. On the basis of a plasma sample volume of $200 \mu \mathrm{L}$, the assay ranged from 2 to $200 \mathrm{ng} / \mathrm{mL}$ for $d$-amphetamine and from 1 to $100 \mathrm{ng} / \mathrm{mL}$ for lisdexamfetamine.

\subsection{Safety Assessments}

Safety evaluations included AEs, vital signs, 12-lead ECGs, physical examination findings, and clinical laboratory parameters. Pulse and blood pressure (BP) were assessed in both supine and standing positions predose (within $30 \mathrm{~min}$ of administration) and at 1.0, 1.5, 2.0, 3.0, 4.0, 6.0, 8.0, 12, 24, 30, 48, and $72 \mathrm{~h}$ after treatment. ECGs were recorded 2,8 , and $72 \mathrm{~h}$ after treatment was administered. TEAEs were defined as AEs that occurred or worsened during the on-treatment period. TEAEs were assigned to the treatment received at the time of onset of the AE. Because this was a crossover study, a reported AE was considered a TEAE for all periods of the crossover up to $72 \mathrm{~h}$ after the last administration within each period, unless a partial date or time showed that it was a TEAE that occurred in an identified period. 
Fig. 1 Treatment regimens. GXR guanfacine extended Regimen A: GXR $4 \mathrm{mg}$ release, $L D X$ lisdexamfetamine dimesylate
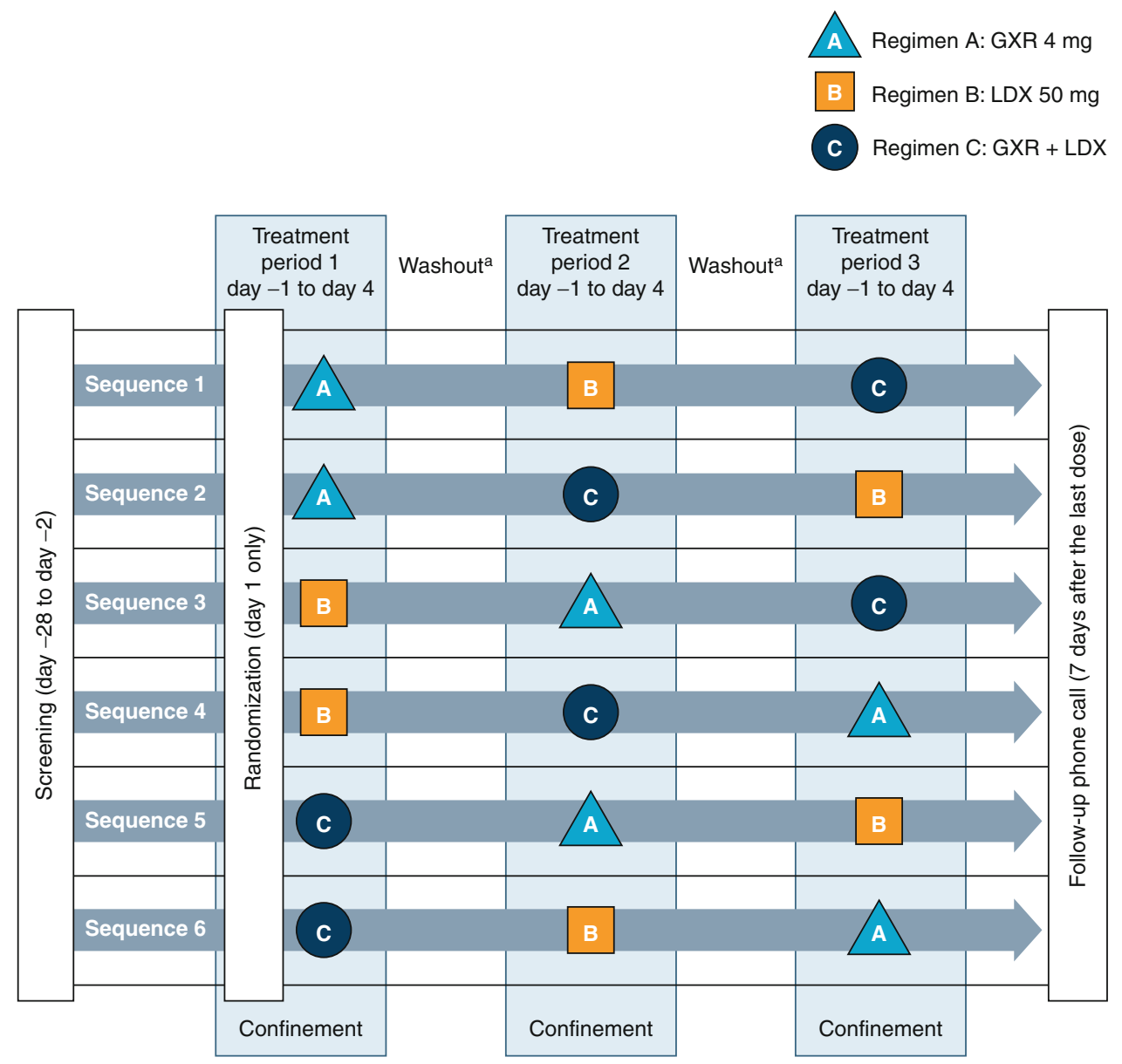

${ }^{a}$ There was to be $a \geq 7$-day washout between the dosing days of the three treatment periods

\subsection{Statistical Analysis}

The primary analysis was the pharmacokinetic analysis performed using data from the pharmacokinetic population. The pharmacokinetic population consisted of all subjects who received at least one dose of the study medication, had at least one postdose safety assessment, and had evaluable concentration-time profiles for guanfacine, LDX, or $d$ amphetamine.

Pharmacokinetic parameters were determined from the plasma concentration-time data by noncompartmental analysis and included the maximum plasma concentration $\left(C_{\max }\right)$, time to $C_{\max }\left(t_{\max }\right)$, area under the plasma concentration-time curve (AUC) to the last measurable concentration at time $t\left(\mathrm{AUC}_{0-t}\right)$, AUC extrapolated to infinity $\left(\mathrm{AUC}_{0-\infty}\right)$, apparent terminal half-life $\left(t_{1 / 2}\right)$, apparent oraldose clearance (CL/F), and apparent volume of distribution $(\mathrm{Vz} / \mathrm{F}) . \mathrm{CL} / \mathrm{F}$ and $\mathrm{Vz} / \mathrm{F}$ were corrected for body weight.

Summary statistics, including the numbers of observations, means with standard deviations (SDs), coefficients of variation, medians, maximums, minimums, and geometric means were determined for all pharmacokinetic parameters for all treatment regimens.

The means of log-transformed pharmacokinetic parameters were compared among (between) treatments using an analysis of variance (ANOVA) with sequence, period, and treatment as fixed effects and subject nested within sequence as a random effect for a crossover study design. To estimate the magnitude of the treatment differences in $C_{\max }$ and $\mathrm{AUC}_{0-\infty}$, the geometric mean ratio (GMR, defined as the least squares mean difference in the logtransformed parameters back-transformed to the original scale) and their $90 \%$ confidence intervals (CIs) were also calculated.

If the $90 \%$ CIs of the GMR ([GXR + LDX]/GXR or $[\mathrm{GXR}+\mathrm{LDX}] / \mathrm{LDX})$ of guanfacine or LDX following coadministration of GXR and LDX to the same analyte following GXR or LDX alone were to fall within the reference interval (0.80-1.25), then the hypothesis of a DDI of GXR and LDX would be rejected. If the CIs were not entirely contained within this interval, then the clinical significance of such mean ratio estimates and confidence 
limits would be interpreted within the context of the therapeutic index.

The available within-subject estimates of the SDs of the log-transformed parameters $\mathrm{AUC}_{0-\infty}(\mathrm{SD}=0.26)$ and $C_{\max }(\mathrm{SD}=0.31)$ for GXR were pooled from previous studies of GXR. A previous study of LDX reported a within-subject SD for log-transformed parameters of 0.215 for $C_{\max }$ and 0.195 for $\mathrm{AUC}_{0-\infty}$ [22]. A total of 36 subjects (six per sequence) were required to demonstrate equivalence, using the bioequivalence reference interval $(0.80-1.25)$, allowing for a $5 \%$ difference between treatment means, to achieve $90 \%$ power.

\section{Results}

\subsection{Subject Disposition and Demographics}

Forty-two subjects were randomized, and 40 (95.2\%) completed the study. No subjects withdrew because of an AE. Early terminations included one withdrawal by a subject and one withdrawal by the primary investigator prior to administration of GXR alone, because of poor tolerance of coadministered GXR and LDX. Demographic and baseline characteristics are summarized in Table 1 .

\subsection{Pharmacokinetic Results}

A summary of the pharmacokinetic parameters of guanfacine and $d$-amphetamine following administration of GXR alone, LDX alone, and GXR and LDX in combination is presented in Table 2.

\subsubsection{Results for Guanfacine}

The mean guanfacine plasma concentration following administration of GXR alone was slightly lower than the mean guanfacine concentration following coadministration with LDX (Fig. 2). Maximum plasma concentrations of guanfacine were attained at a median of $6 \mathrm{~h}$ after administration of GXR alone or in combination with LDX. The $90 \% \mathrm{CI}$ of the GMR of $\mathrm{AUC}_{0-\infty}$ for guanfacine following GXR administered alone and in combination with LDX was $0.981-1.162$ and met strict bioequivalence criteria requiring $90 \%$ CIs to fall within the interval of $0.80-1.25$. The $90 \% \mathrm{CI}$ of the GMR of $C_{\max }$ for guanfacine following administration of GXR alone and in combination with LDX was 1.066-1.321 and did not fall within the standard bioequivalence reference interval. The upper bound of the $90 \% \mathrm{CI}$ of $C_{\max }$ for guanfacine exceeded the standard range for bioequivalence by $7 \%$ when GXR was coadministered with LDX.
Table 1 Summary of demographic and baseline characteristics of the study population $(N=42)^{\mathrm{a}}$

\begin{tabular}{|c|c|}
\hline Characteristic & Value \\
\hline \multicolumn{2}{|l|}{ Age (years) } \\
\hline Mean [SD] & $30.5[7.41]$ \\
\hline Median & 28.5 \\
\hline Minimum, maximum & 18,45 \\
\hline \multicolumn{2}{|l|}{$\operatorname{Sex}(n[\%])$} \\
\hline Male & $33[78.6]$ \\
\hline Female & 9 [21.4] \\
\hline \multicolumn{2}{|l|}{ Body weight (kg) } \\
\hline Mean [SD] & $78.2[11.20]$ \\
\hline Median & 75.6 \\
\hline Minimum, maximum & 54,101 \\
\hline \multicolumn{2}{|l|}{ Height $(\mathrm{cm})$} \\
\hline Mean [SD] & $173.8[8.76]$ \\
\hline Median & 175.5 \\
\hline Minimum, maximum & 157,189 \\
\hline \multicolumn{2}{|l|}{ Body mass index $\left(\mathrm{kg} / \mathrm{m}^{2}\right)$} \\
\hline Mean [SD] & $25.8[2.55]$ \\
\hline Median & 25.9 \\
\hline Minimum, maximum & 21,30 \\
\hline \multicolumn{2}{|l|}{ Ethnicity $(n[\%])$} \\
\hline Hispanic or Latino & 12 [28.6] \\
\hline Not Hispanic or Latino & $30[71.4]$ \\
\hline \multicolumn{2}{|l|}{ Race $(n[\%])$} \\
\hline White & 15 [35.7] \\
\hline Black or African American & 27 [64.3] \\
\hline
\end{tabular}

\subsubsection{Results for d-Amphetamine and Lisdexamfetamine}

The mean $d$-amphetamine plasma concentrations following administration of LDX alone were essentially identical to those following coadministration with GXR (Fig. 3a). Maximum plasma concentrations of $d$-amphetamine were attained at a median of $4 \mathrm{~h}$ following dosing of LDX alone or in combination with GXR. The $90 \%$ CIs of the GMRs for $C_{\max }$ and $\mathrm{AUC}_{0-\infty}$ for $d$-amphetamine following administration of LDX alone and in combination with GXR (0.967-1.019 and 0.983-1.06, respectively) met strict bioequivalence criteria requiring $90 \%$ CIs to fall within the interval of $0.80-1.25$.

Similar profiles for mean plasma LDX concentrations were obtained for regimen B (LDX alone) and regimen C (LDX and GXR) (Fig. 3b). When LDX was given alone and in combination with GXR, its mean maximum concentrations were 26.14 and $27.13 \mathrm{ng} / \mathrm{mL}$, respectively, and were obtained at $1.1 \mathrm{~h}$. 
Table 2 Pharmacokinetic parameters of guanfacine and $d$-amphetamine

\begin{tabular}{|c|c|c|c|c|c|c|}
\hline Parameter & $C_{\max }(\mathrm{ng} / \mathrm{mL})$ & $t_{\max }(\mathrm{h})$ & $\operatorname{AUC}_{0-\infty}(\mathrm{ng} \cdot \mathrm{h} / \mathrm{mL})$ & $t_{1 / 2}(\mathrm{~h})$ & $\mathrm{CL} / \mathrm{F}(\mathrm{L} / \mathrm{h} / \mathrm{kg})$ & $\mathrm{Vz} / \mathrm{F}(\mathrm{L} / \mathrm{kg})$ \\
\hline \multicolumn{7}{|c|}{ Summary of guanfacine pharmacokinetic parameters } \\
\hline \multicolumn{7}{|l|}{ GXR alone } \\
\hline$N$ & 40 & 40 & 37 & 37 & 37 & 37 \\
\hline Mean $[S D]$ & $2.55[1.03]$ & $8.6[7.7]$ & $104.9[34.7]$ & $23.5[10.2]$ & $0.54[0.17]$ & $17.36[7.54]$ \\
\hline Median & 2.30 & 6 & 102.4 & 20.5 & 0.51 & 15.34 \\
\hline Minimum, maximum & $0.98,5.79$ & $1.5,30$ & $54,218.2$ & $11.4,50$ & $0.27,1.04$ & $7.02,38.05$ \\
\hline \multicolumn{7}{|l|}{$\mathrm{GXR}+\mathrm{LDX}$} \\
\hline$N$ & 41 & 41 & 39 & 39 & 39 & 39 \\
\hline Mean $[\mathrm{SD}]$ & $2.97[0.98]$ & $7.9[5]$ & $112.8[35.7]$ & $21.4[8.2]$ & $0.5[0.15]$ & $15.33[7.35]$ \\
\hline Median & 2.87 & 6 & 109.4 & 18.8 & 0.46 & 13.61 \\
\hline Minimum, maximum & $1.52,5.60$ & 3,30 & $61.5,213.6$ & $11.9,48.2$ & $0.3,0.89$ & $6.36,44.79$ \\
\hline \multicolumn{7}{|c|}{ Summary of $d$-amphetamine pharmacokinetic parameters } \\
\hline \multicolumn{7}{|l|}{ LDX alone } \\
\hline$N$ & 41 & 41 & 41 & 41 & 41 & 41 \\
\hline Mean [SD] & $36.48[7.13]$ & $4.2[1.1]$ & $686.9[159.8]$ & $11.2[1.6]$ & $0.99[0.23]$ & $15.58[2.52]$ \\
\hline Median & 36.95 & 4 & 687.7 & 11.3 & 0.93 & 15.33 \\
\hline Minimum, maximum & $20.51,57.15$ & 3,6 & $324.6,1070$ & $8.3,14.6$ & $0.66,1.8$ & $11.16,21.77$ \\
\hline \multicolumn{7}{|l|}{ GXR + LDX } \\
\hline$N$ & 41 & 41 & 41 & 41 & 41 & 41 \\
\hline Mean $[S D]$ & $36.50[6.00]$ & $3.9[1.1]$ & $708.4[137.8]$ & $11.2[1.5]$ & $0.95[0.17]$ & $15.11[2.37]$ \\
\hline Median & 35.71 & 4 & 713.6 & 11 & 0.95 & 14.43 \\
\hline Minimum, maximum & $23.05,53.06$ & 3,8 & $456.1,954.1$ & $8,15.1$ & $0.67,1.34$ & $11.45,23.8$ \\
\hline
\end{tabular}

$A U C_{0-\infty}$ area under the plasma concentration-time curve extrapolated to infinity, $C L / F$ apparent oral-dose clearance, $C_{\max }$ maximum plasma concentration, GXR guanfacine extended release, $L D X$ lisdexamfetamine dimesylate, $S D$ standard deviation, $t_{1 / 2}$ apparent terminal half-life, $t_{m a x}$ time to maximum plasma concentration, $V z / F$ apparent volume of distribution

Fig. 2 Mean guanfacine plasma concentrations over time following administration of guanfacine extended release (GXR) alone and in combination with lisdexamfetamine dimesylate (LDX). A time shift has been applied to the figure; values have been slightly staggered on the $\mathrm{x}$-axis for clarity, as some values were similar between the two treatment regimens

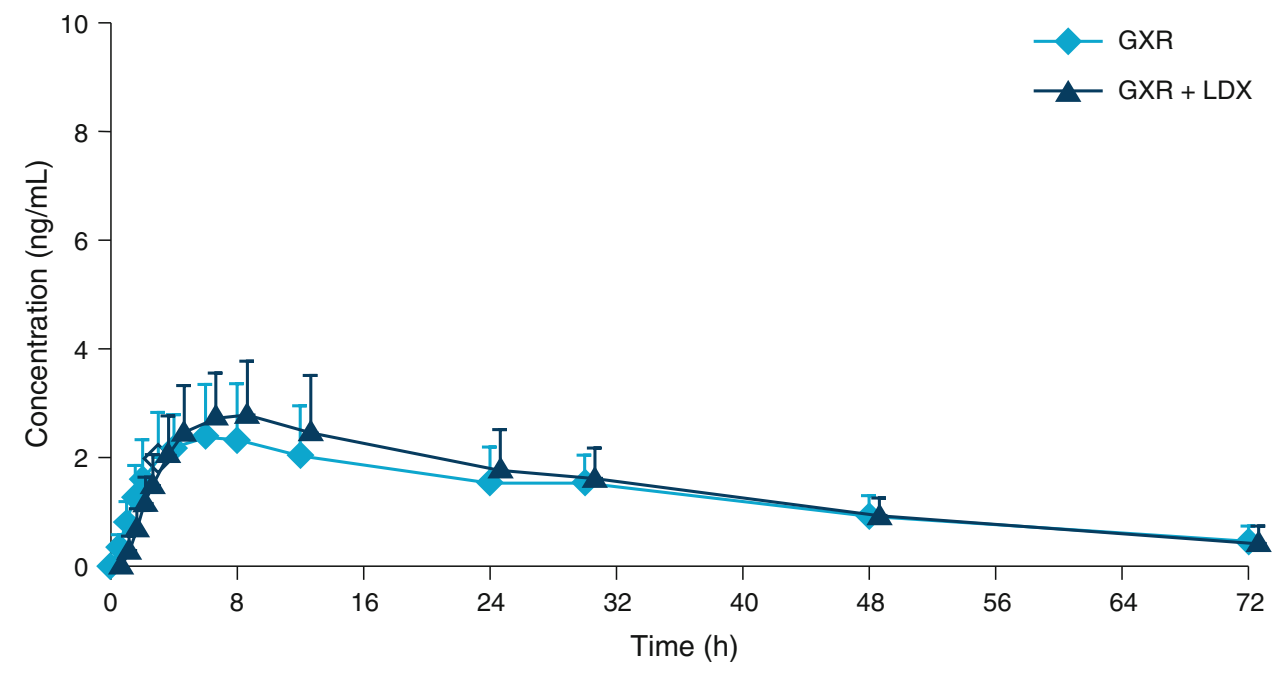

\subsection{Safety Results}

\subsubsection{Treatment-Emergent Adverse Events}

A total of 18 subjects (42.9\%) reported at least one TEAE. TEAEs were reported in seven subjects $(17.5 \%)$, eight subjects (19.5\%), and 10 subjects $(24.4 \%)$ while they were receiving GXR, LDX, and GXR and LDX in combination, respectively. The most commonly reported individual TEAEs (occurring in $\geq 5 \%$ of subjects during any regimen) were dizziness (5.0, 7.3, and $7.3 \%$ ), postural dizziness $(10.0,2.4$, and $0 \%)$, and headache $(7.5,4.9$, and 
Fig. 3 a Mean $d$-amphetamine plasma concentrations and b mean lisdexamfetamine dimesylate (LDX) plasma concentrations over time following administration of LDX alone and in combination with guanfacine extended release (GXR). A time shift has been applied to the figure; values have been slightly staggered on the $\mathrm{x}$-axes for clarity, as some values were similar between the two treatment regimens

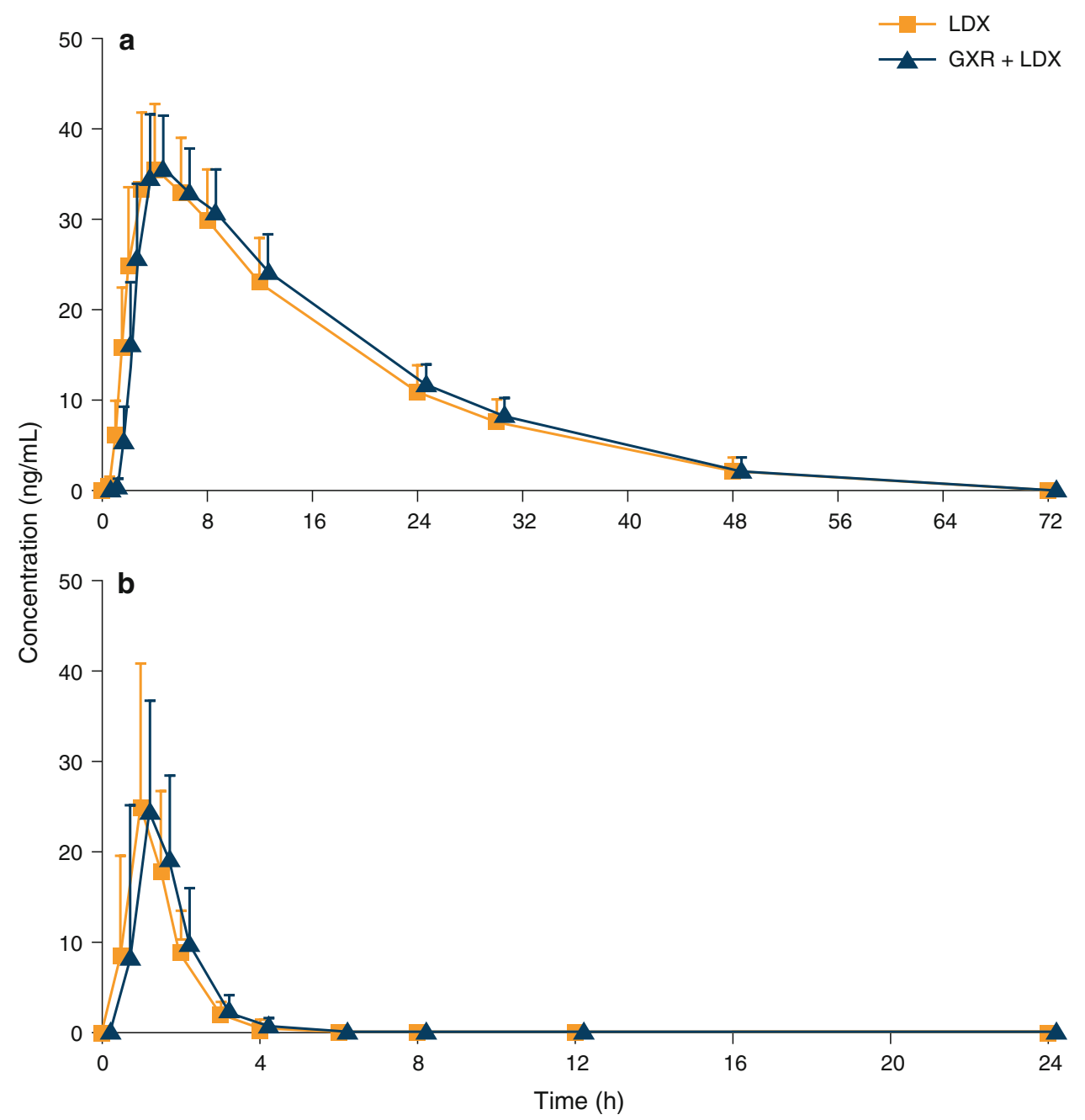

$7.3 \%$ ) following administration of GXR, LDX, and GXR and LDX in combination, respectively. Most TEAEs were mild. No discontinuations were due to TEAEs.

\subsubsection{Vital Signs}

The supine pulse rate, SBP, and DBP following administration of GXR alone and LDX alone were similar to those previously observed for either drug. Following administration of GXR alone, there was a modest decrease in pulse rate, which began to return toward predose levels after hour 6. Supine SBP and DBP were modestly decreased across the 12-h period. Following administration of LDX alone, there was a modest increase in pulse rate, as well as increases in supine SBP and DBP.

Coadministration of LDX and GXR yielded results similar to those seen with LDX administered alone, such that a modest increase in supine pulse rate, as well as increases in SBP and DBP, was observed following coadministration (Figs. 4, 5). There did not appear to be clinically important differences in postural orthostatic changes (i.e., differences between standing and supine parameters) in pulse rate or in BP following coadministration of GXR and LDX compared with GXR alone.

\subsubsection{Electrocardiogram Results}

Overall, clinically meaningful changes in ECGs were not observed, and the ECG results for GXR alone and LDX alone were consistent with the known effects of these medications. Two subjects had clinically significant abnormalities in ECG results. One subject had a wandering atrial pacemaker $2 \mathrm{~h}$ after administration of LDX. The subject was asymptomatic, and the event was considered mild and resolved the same day. The other subject had a supraventricular arrhythmia (first-degree atrioventricular block [pulse rate interval $=204 \mathrm{~ms}$ ] with bradycardia [45 beats/min] and escape beats) $2 \mathrm{~h}$ after coadministration. The subject was asymptomatic, and the event was considered mild and resolved the next day. 
Fig. 4 Mean ( \pm standard deviation) supine pulse rate over hours 1 to 12 following study drug administration (observed values). GXR guanfacine extended release, $L D X$ lisdexamfetamine dimesylate
Fig. 5 a Mean ( \pm standard deviation [SD]) supine systolic blood pressure (SBP) and b mean $( \pm \mathrm{SD})$ supine diastolic blood pressure (DBP) over hours 1 to 12 following study drug administration (observed values). GXR guanfacine extended release, $L D X$ lisdexamfetamine dimesylate
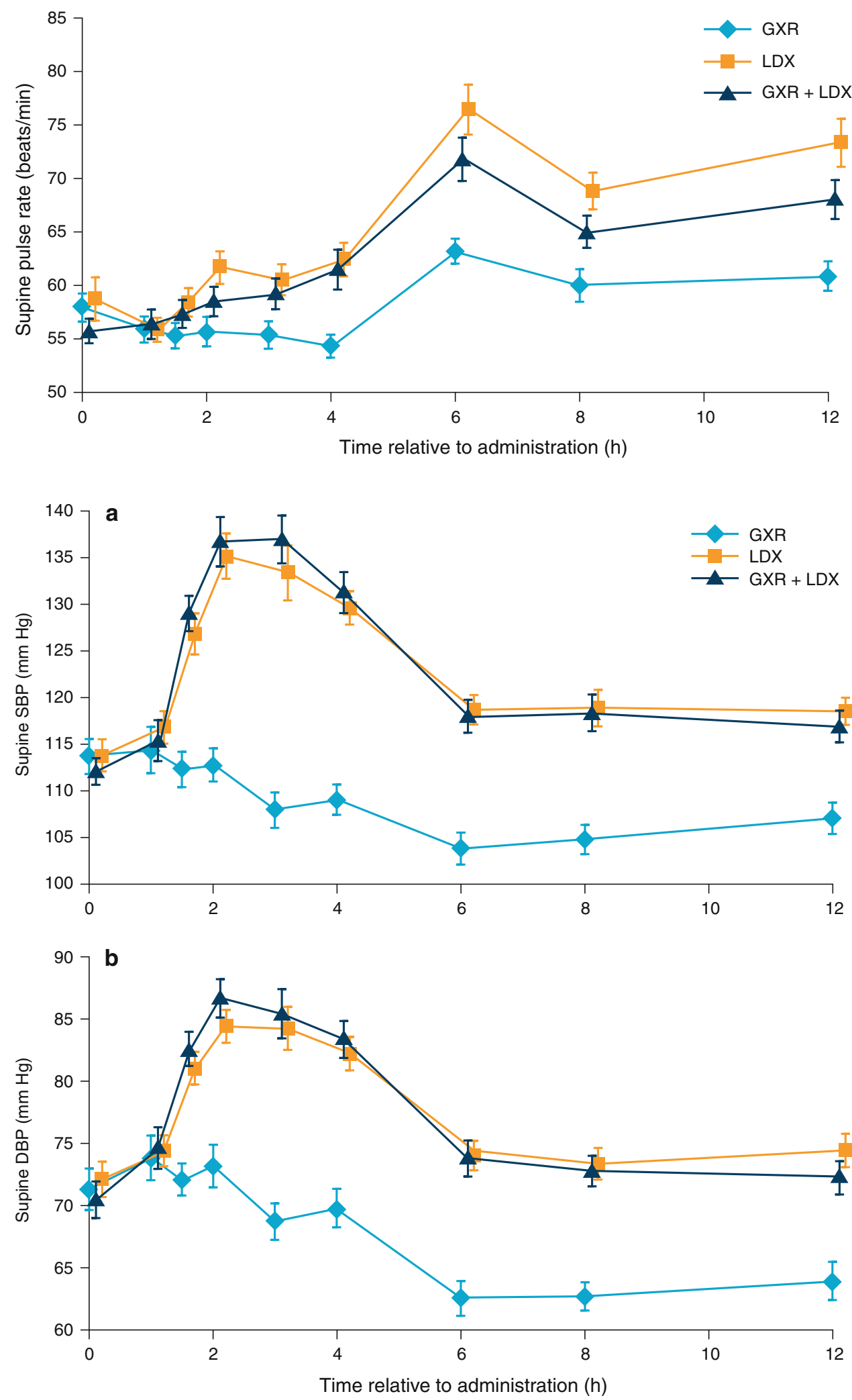

\section{Discussion}

Guanfacine is known to be metabolized by CYP3A4 [5]. While intact LDX is not metabolized by the CYP system and is neither an inducer nor an inhibitor of the system, the metabolism of amphetamine has not been fully elucidated [18]. Data have suggested that CYP2D6 is involved in the metabolism of amphetamine, and in vitro studies have 
suggested that amphetamine and its metabolites inhibit CYP2D6, CYP1A2, and CYP3A4 enzymes [13, 18, 2729]. Therefore, it was prudent to evaluate the pharmacokinetics of GXR coadministered with LDX to confirm a lack of metabolic interactions between these two medications, as GXR is likely to be adjunctively administered with psychostimulants such as LDX to treat ADHD.

Although the guanfacine $C_{\max }$ exceeded the standard upper limit of bioequivalence by $7 \%$ when GXR was coadministered with LDX, overall, coadministration of GXR and LDX did not result in a clinically meaningful pharmacokinetic DDI compared with the pharmacokinetics of either treatment administered alone. The $90 \%$ CIs of the GMRs for $\mathrm{AUC}_{t}$ and $\mathrm{AUC}_{0-\infty}$ for guanfacine following administration of GXR alone and in combination with LDX fell within the reference interval $(0.80-1.25)$. The guanfacine $C_{\max }$ was increased by $19 \%$ when GXR was coadministered with LDX. The $90 \%$ CIs of the GMRs for $C_{\max }, \mathrm{AUC}_{t}$, and $\mathrm{AUC}_{0-\infty}$ for $d$-amphetamine following administration of LDX alone and in combination with GXR fell entirely within the reference interval (0.80-1.25).

The TEAEs reported in this study were expected and were consistent with those observed historically with psychostimulants administered alone or with GXR [5-7, 30, 31]. No differences in the type, incidence, or severity of TEAEs among treatment groups were observed, and no subject discontinued treatment because of an AE. In addition, no clinically meaningful changes in ECGs, clinical laboratory parameters, or physical examinations were noted during the study.

\subsection{Study Limitations}

The results of this small open-label study, conducted in a medically healthy adult population, should be viewed with consideration of several limitations. As GXR is approved for the treatment of ADHD in children and adolescents aged 6-17 years [5], the healthy adult subjects in this study may not have been representative of the population commonly treated with this medication in a clinical setting. In addition to age considerations, more studies would be needed to determine if similar outcomes would be seen in populations likely to receive adjunctive administration in clinical practice (e.g., subjects with comorbid disorders). In addition, subjects with comorbidities that may contribute to cardiac AEs were excluded from the study.

Caution should also be used in interpreting these results, as this study was designed to assess the pharmacokinetic parameters of coadministration of GXR and LDX; the study was not designed to robustly assess the cardiovascular effects of coadministration. As this was a single-dose rather than multiple-dose study, the effects that were observed may not be representative of those occurring at steady state. Therefore, the findings of this study may not be readily extrapolated to the therapeutic setting.

Finally, it is not known if similar safety and cardiovascular effects would be seen in large, randomized, double-blind, placebo-controlled studies, or in studies that assessed coadministration of GXR and LDX over a longer time period. Future studies should examine these areas, as well as the efficacy of coadministration.

\section{Conclusions}

Overall, coadministration of GXR and LDX did not result in a clinically meaningful pharmacokinetic DDI compared with the pharmacokinetics of either treatment administered alone. No unique TEAEs were observed with coadministration of GXR and LDX compared with either treatment alone. However, as the study was not designed to robustly assess cardiovascular effects and other safety parameters, further study of the safety of coadministration of GXR and psychostimulants is warranted.

Acknowledgments With great sadness, the authors wish to acknowledge the passing of our colleague, Mary Haffey, and recognize her contributions to this article.

Funding and Individual Contributions This clinical research was funded by the sponsor, Shire Development LLC (Wayne, PA, USA). Under direction from the authors, Jennifer Steeber $\mathrm{PhD}$ [an employee of SCI Scientific Communications \& Information (SCI); Parsippany, NJ, USA] provided writing assistance for this publication. Editorial assistance in the form of proofreading, copy editing, and fact checking was also provided by SCI. Jonathan Rubin MD MBA, Carla White BSc CStat, Edward Johnson, Michael Kahn, and Gina D'Angelo PharmD MBA, from Shire Development LLC, and Sharon Youcha MD [a former employee of Shire Development LLC] also reviewed and edited the manuscript for scientific accuracy. Additional editorial support was provided by Wilson Joe, PhD, of MedErgy (Yardley, PA, USA). Shire Development LLC provided funding to SCI and MedErgy for support in writing and editing this manuscript. Although the sponsor was involved in the design, collection, analysis, interpretation, and fact checking of information, the content of this manuscript, the ultimate interpretation, the accuracy of the study results, and the decision to submit it for publication in Drugs in $R \& D$ was made by the authors independently.

Conflict of Interest Disclosures Benno Roesch is affiliated with Advanced Biomedical Research, Inc. (Hackensack, NJ, USA). Mary Corcoran, Jaideep Purkayastha, Philip Wang, and James Ermer are employees of Shire and hold stock and/or stock options in Shire. Jennifer Fetterolf and Peter Preston are consultants of Shire. Mary Haffey was an employee of Shire and held stock and/or stock options in Shire. Patrick Martin is an employee of Shire.

Open Access This article is distributed under the terms of the Creative Commons Attribution Noncommercial License which permits any noncommercial use, distribution, and reproduction in any medium, provided the original author(s) and the source are credited. The exclusive right to any commercial use of the article is with Springer. 


\section{References}

1. Adler LA, Reingold LS, Morrill MS, et al. Combination pharmacotherapy for adult ADHD. Curr Psychiatry Rep. 2006;8(5): 409-15.

2. Popper CW. Combining methylphenidate and clonidine: pharmacologic questions and news reports about sudden death. J Child Adolesc Psychopharmacol. 1995;5(3):157-66.

3. Brown TE. Atomoxetine and stimulants in combination for treatment of attention deficit hyperactivity disorder: four case reports. J Child Adolesc Psychopharmacol. 2004;14(1):129-36.

4. Pohl GM, Van Brunt DL, Ye W, et al. A retrospective claims analysis of combination therapy in the treatment of adult attention-deficit/hyperactivity disorder (ADHD). BMC Health Serv Res. 2009;9:95

5. Intuniv (package insert). Wayne: Shire Pharmaceuticals Inc.; 2011.

6. Biederman J, Melmed RD, Patel A, et al., for the SPD503 Study Group. A randomized, double-blind, placebo-controlled study of guanfacine extended release in children and adolescents with attention-deficit/hyperactivity disorder. Pediatrics. 2008;121(1): e73-e84.

7. Sallee F, McGough J, Wigal T, et al., for the SPD503 Study Group. Guanfacine extended release in children and adolescents with attention-deficit/hyperactivity disorder: a placebo-controlled trial. J Am Acad Child Adolesc Psychiatry. 2009;48(2):155-65.

8. Sallee FR, Lyne A, Wigal T, et al. Long-term safety and efficacy of guanfacine extended release in children and adolescents with attention-deficit/hyperactivity disorder. J Child Adolesc Psychopharmacol. 2009;19(3):215-26.

9. Biederman J, Melmed RD, Patel A, et al. Long-term, open-label extension study of guanfacine extended release in children and adolescents with ADHD. CNS Spectr. 2008;13(12):1047-55.

10. Connor DF, Findling RL, Kollins SH, et al. Effects of guanfacine extended release on oppositional symptoms in children aged 6-12 years with attention-deficit hyperactivity disorder and oppositional symptoms: a randomized, double-blind, placebo-controlled trial. CNS Drugs. 2010;24(9):755-68.

11. Faraone SV, Pucci M, Coghill D. Pharmacotherapy for attentiondeficit-hyperactivity disorder. US Psychiatry Rev. 2009;2(1): $17-27$.

12. Wilens TE, Spencer TJ. Understanding attention-deficit/hyperactivity disorder from childhood to adulthood. Postgrad Med. 2010;122(5):97-109.

13. Vyvanse (package insert). Wayne: Shire US Inc.; 2012.

14. Spencer TJ, Greenbaum M, Ginsberg LD, et al. Safety and effectiveness of coadministration of guanfacine extended release and psychostimulants in children and adolescents with attentiondeficit/hyperactivity disorder. J Child Adolesc Psychopharmacol. 2009;19(5):501-10.

15. Wilens TE, Youcha S, Lyne A, et al. A multisite placebo-controlled trial of morning or evening dosed extended-release guanfacine in combination with psychostimulants in children and adolescents with ADHD. 65th Annual Meeting of the Society of Biological Psychiatry; 2010 May 20-22; New Orleans.

16. US Department of Health and Human Services. Guidance for industry: in vivo drug metabolism/drug interaction studies- study design, data analysis, and recommendations for dosing and labeling. http://www.fda.gov/downloads/Drugs/Guidance ComplianceRegulatoryInformation/Guidances/ucm072119.pdf. Accessed 26 Oct 2012.

17. Pennick M. Absorption of lisdexamfetamine dimesylate and its enzymatic conversion to $d$-amphetamine. Neuropsychiatr Dis Treat. 2010;6(1):317-27.

18. Krishnan S, Moncrief S. An evaluation of the cytochrome p450 inhibition potential of lisdexamfetamine in human liver microsomes. Drug Metab Dispos. 2007;35(1):180-4.

19. Boellner SW, Pennick M, Fiske K, et al. Pharmacokinetics of a guanfacine extended-release formulation in children and adolescents with attention-deficit-hyperactivity disorder. Pharmacotherapy. 2007;27(9):1253-62.

20. Swearingen D, Pennick M, Shojaei A, et al. A phase I, randomized, open-label, crossover study of the single-dose pharmacokinetic properties of guanfacine extended-release 1-, 2-, and 4-mg tablets in healthy adults. Clin Ther. 2007;29(4):617-25.

21. Boellner SW, Stark JG, Krishnan S, et al. Pharmacokinetics of lisdexamfetamine dimesylate and its active metabolite, $d$ amphetamine, with increasing oral doses of lisdexamfetamine dimesylate in children with attention-deficit/hyperactivity disorder: a single-dose, randomized, open-label, crossover study. Clin Ther. 2010;32(2):252-64.

22. Ermer J, Homolka R, Martin P, Purkayastha J, et al. Lisdexamfetamine dimesylate: linear dose-proportionality, low intersubject and intrasubject variability, and safety in an open-label single-dose pharmacokinetic study in healthy adult volunteers. J Clin Pharmacol. 2010;50(9):1001-10.

23. Krishnan SM, Pennick M, Stark JG. Metabolism, distribution and elimination of lisdexamfetamine dimesylate: open-label, singlecentre, phase I study in healthy adult volunteers. Clin Drug Investig. 2008;28(12):745-55.

24. Krishnan SM, Stark JG. Multiple daily-dose pharmacokinetics of lisdexamfetamine dimesylate in healthy adult volunteers. Curr Med Res Opin. 2008;24(1):33-40.

25. Biederman J, Boellner SW, Childress A, et al. Lisdexamfetamine dimesylate and mixed amphetamine salts extended-release in children with ADHD: a double-blind, placebo-controlled, crossover analog classroom study. Biol Psychiatry. 2007;62(9):970-6.

26. Adler LA, Goodman DW, Kollins SH, et al, on behalf of the 303 Study Group. Double-blind, placebo-controlled study of the efficacy and safety of lisdexamfetamine dimesylate in adults with attention-deficit/hyperactivity disorder. J Clin Psychiatry. 2008;69(9): 1364-73.

27. Markowitz JS, Patrick KS. Pharmacokinetic and pharmacodynamic drug interactions in the treatment of attention-deficit hyperactivity disorder. Clin Pharmacokinet. 2001;40(10):753-72.

28. Adderall XR (package insert). Wayne: Shire US Inc.; 2010.

29. Bach MV, Coutts RT, Baker GB. Involvement of CYP2D6 in the in vitro metabolism of amphetamine, two $\mathrm{N}$-alkylamphetamines and their 4-methoxylated derivatives. Xenobiotica. 1999;29(7): 719-32.

30. Wilens TE, Spencer TJ. The stimulants revisited. Child Adolesc Psychiatr Clin N Am. 2000;9(3):573-603, viii.

31. Concerta (package insert). Titusville: McNeil Pediatrics; 2010. 